\title{
Automation Testing In Software Organization
}

\author{
Prasad Mahajan \\ Bharati vidyapeeth $\mathrm{COE}$ \\ Pune, India
}

\author{
Harshal Shedge \\ Bharati vidyapeeth $\mathrm{COE}$ \\ Pune, India
}

\author{
Uday Patkar \\ Bharati vidyapeeth $\mathrm{COE}$ \\ Pune, India
}

\begin{abstract}
In software testing, automation testing plays a great role for improving test efficiency of the software testing team. Sometimes manual testing may not be effective due to its inconsistency, lack of coverage and none repeating in nature. To overcome this Test automation is used in software industry. In this paper we will discuss about Test Automation its pre-requisites, working steps, when to use automation testing, benefits over manual testing and selection of test cases to automate Thus there are a number of testing tools available in the market out of which we will also discuss about Selenium automation tool
\end{abstract}

Keywords: Test automation, Testing tools, Automation testing, Test case, Manual testing, Pre-requisites, Selenium

\section{INTRODUCTION}

Software testing is a set of activities conducted for finding errors in software. It is a process used to measure the quality of the software. Manual testing and automation testing are the two ways of testing. Manual testing is also called as static testing. It is carried out by the tester. Automation testing is also called as dynamic testing. But the problem is it is very time consuming process and requires more effort. So, automation testing is used to solve these problems. Automated testing is divided into four types such as reliability testing, security testing, correctness testing, and performance testing. It automates the steps of manual testing using automation tools. Automated tests are fast to execute and they are repeatable in nature. There are various tools available in the market which are used to test the process and targeted specific test environment. The environment may be functional, performance or exceptional testing etc. Testing tool should be selected on the basis of its compatibility with checklist for that purpose pilot round of the corresponding tool should be done. Cost is also an important factor for selection of tool.

\section{AUTOMATION TESTING}

\subsection{Test Automation stages[3]}

Please Normally the Automation manager will be responsible for selection of tool, Test engineer will be responsible for script generation, deployment and execution this stage will decide whether we need to automate the project or not. If failure occurs in this stage then it creates a larger impact on the project execution.

Following figure shows various stages of a test automation process.

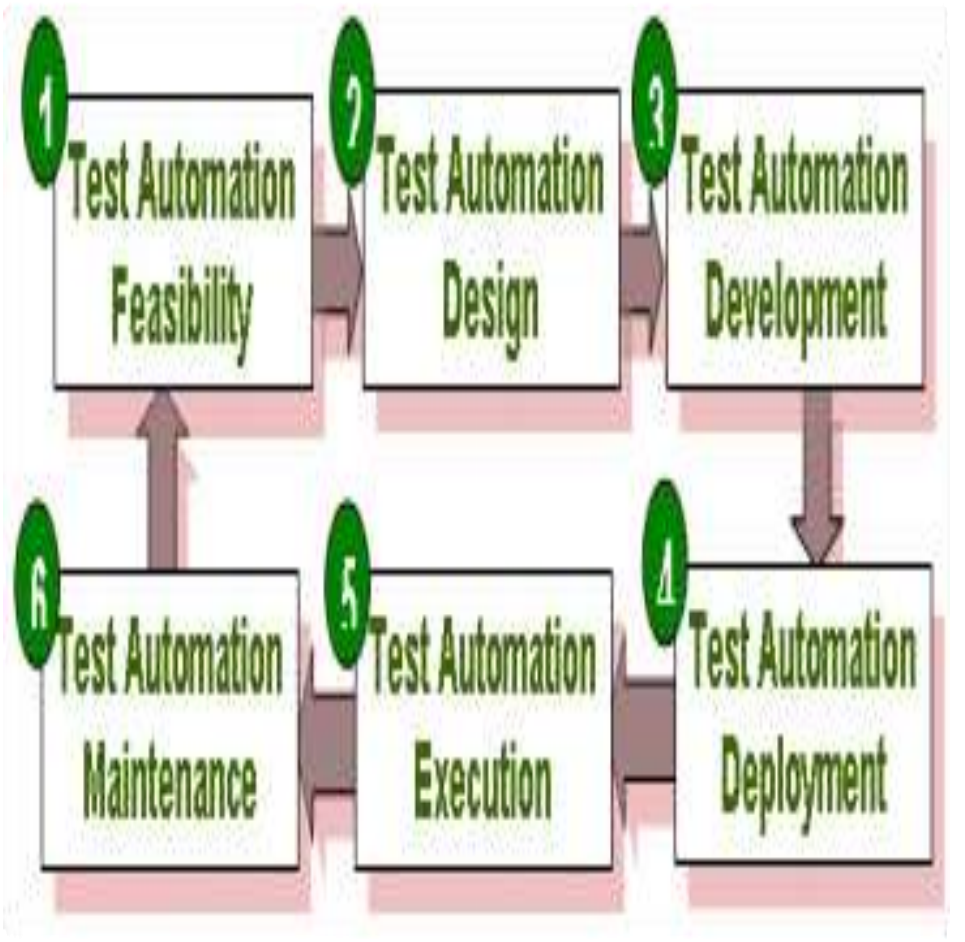

Figure1. Stages of Automation 


\subsection{When to Automate [3]}

1. After all manual testing process is in place as per expected results

\section{At least tested twice}

3. Environmental/Build should be constant or stable

\subsection{Benefits over manual testing [2]}

Following are some of the benefits of test automation

1. Fast application development by reducing time for testing

2. Repeatable in nature

3. Reliable

4. Scripts are reusable

5. Programmable

6. Provides high coverage for regression testing without adding additional resources

7. Improvement in productivity

8. Detailed test logs

9. Execution of the scripts across multiple platforms

10. Cost effectiveness improvement

\subsection{Test cases to automate [3]}

Selection of correct test cases for automation is very important as it impacts on development cost and scheduled time. One time testing is not done by automation tool. Also automation testing is not use for Usability testing to check "How easy is the web site to use?". This testing technique is not useful for tests which don't have predictable results.

Tests that need to be run for every build should be selected for automation.

Test that uses multiple data values for the same action are also needed for automation.

Similar tests that need to be executed using different platforms.

Regression testing is done by automation tool.

\subsection{Applications [1]}

Automation testing is use for testing web based application for finding flaws in the application and also to check the security of the system.
It provides a complimentary approach to manual testing to use automation testing to improve the level of test automation and reduction of risk instead of using more people.

For test management automation testing provides different types of tools.

Effective defect tracking is done by automation tool.

Table1. Automation Testing Vs Manual Testing

\begin{tabular}{|c|c|c|}
\hline $\begin{array}{c}\text { Information } \\
\text { types }\end{array}$ & Automation testing & Manual testing \\
\hline $\begin{array}{c}\text { Execution } \\
\text { speed }\end{array}$ & Fast to execute & $\begin{array}{c}\text { Slower than } \\
\text { automation } \\
\text { testing }\end{array}$ \\
\hline Sequence & $\begin{array}{c}\text { After manual } \\
\text { testing }\end{array}$ & $\begin{array}{c}\text { Before } \\
\text { automation } \\
\text { testing }\end{array}$ \\
\hline Resources & $\begin{array}{c}\text { Tool should be } \\
\text { used instead of } \\
\text { large number of } \\
\text { human resource }\end{array}$ & $\begin{array}{c}\text { Large number of } \\
\text { human resource is } \\
\text { required }\end{array}$ \\
\hline
\end{tabular}




\section{SOFTWARE TESTING TOOLS [2]}

\subsection{Selenium}

Selenium is an open source tool.

It is a robust set of tools that supports rapid development of test automation for web-based applications. Selenium Supports Cross Browser Testing as well as selenium tests can be run on multiple browsers.

It allows scripting in several languages like Java, C\#, PHP and Python.

Assertion statements in selenium provide an efficient way of comparing expected and actual results.

\section{Selenium consist of following components}

1. Selenium IDE

2. Selenium RC

3. Selenium Grid

\subsection{Selenium IDE}

- Selenium IDE is an integrated development environment for Selenium tests. It is implemented as a Firefox extension, and allows you to record, edit, and playback the test. Selenium IDE

- Provides a way to save tests as Java, Ruby scripts, HTML or any other format.

- To use selenium tools first we have to install it

- Using Firefox, first download the IDE from the Selenium HQ downloads page then Restart Firefox. After that you will find the Selenium-IDE listed under the Firefox Tools menu.

Selenium allows you to

- To set the Text Encoding Format

- Setting of Default Page Time Out

- Provides the Base URL recording option

- $\quad$ Adding Selenium Core and IDE Extension

\subsection{Procedure of creating test cases [5]}

- For recording and playing test cases first

- Open Firefox which has IDE installed

- Open the application to record.

- Go To Tools $\rightarrow$ and opened the Selenium IDE

- Now perform the various operations on the application as you are testing the application.

- Once your recording is done click on the stop recording button and save the test case through the file menu. By default it will be saved in HTML format.

- To play the recorded test Click on the run button which is present on the user interface and you can also manage the speed of the execution.

- Once test execution is over you can view the test result in the bottom of the IDE window

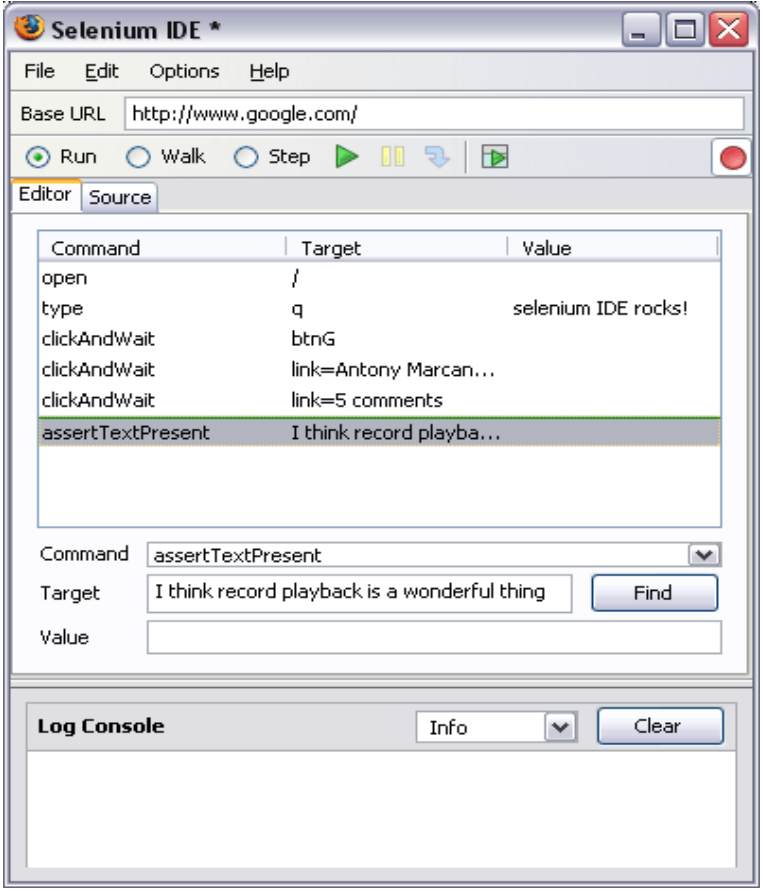

Figure1 Selenium IDE-UI

\subsection{Selenium RC (Remote Control)}

- Selenium RC is a solution to cross browser testing.

- It is a server which is written in Java and available on all the platforms.

\subsection{Selenium Grid}

- Selenium-Grid allows the test suites to be run in multiple environments.

- Multiple instances of Selenium-RC are running on various operating system and browser configurations with the help of selenium-grid.

\section{Conclusion}

- Test automation process is use to minimize the cost and other overheads. Automation results in reduction of time spend on regression tests and provide an opportunity for organizations to improve the quality of their software products. Because automation tool can perform test faster than human.

- But effectiveness of automation testing depends on selection of appropriate tool which is compatible with checklist, selection of test cases to automate. Cost is also an important factor considered for selection of tool.

- Selenium tests is use to check workflow but it cannot be put in development build and it cannot be considered as replacement for exploratory testing 


\section{ACKNOWLEDGMENTS}

We are very thankful to uday patkar who have contributed towards development of this template.. and other teaching staff who helps in the development of this paper

\section{REFERENCES}

[1] The Application of Software Testing Technology on Security in Web Application System" ISSN: 1662:7482 Vals-556-562 Department of information Engineering, Henan polytechnic China 450046.

[2] Research on Software Testing Tools Rasneet Kaur Chauhan $\dot{A}^{*}$ and Iqbal SinghB $\dot{A}$ Department of Computer Science India
[3] Software Test Automation Abdul Rauf EMa,*, E.Madhusudhana Reddyb aResearch and Development Centre ,Bharathiyar University,Coimbatore-641014,

[4] Achievements for software testing Mark Harman, Yue Jia and Yuanyuan ZhangUniversity College London, CREST Centre, London, UK

[5] Software Testing Research and Practice ISTI-CNR Italy 